\title{
Case report of a piece of dishwashing steel wool in a child's pharynx
}

\author{
Parvin Abbaslou, Maryam Ahmadipour* \\ Department of Pediatric, Faculty of Medicine, Kerman University of Medical Sciences, Kerman, Iran
}

\author{
Received: 13 February 2020 \\ Accepted: 1 April 2020 \\ Published online: 8 April 2020 \\ *Corresponding author: \\ Maryam Ahmadipour, \\ maryam.ahmadipour5@gmail.com \\ Competing interests: None. \\ Funding information: None. \\ Citation: Abbaslou P, Ahmadipour \\ M. Case report of a piece of \\ dishwashing steel wool in a child's \\ pharynx. Journal of Emergency \\ Practice and Trauma 2020; 6(2): 118 \\ 120. doi: 10.34172/jept.2020.09.
}

\begin{abstract}
Objective: Foreign body swallowing is a common pediatric problem. A foreign body in the pharynx is a medical emergency that requires urgent intervention. Evaluation and treatment of pharyngeal foreign bodies is much more difficult in children than in adults and sometimes requires hospitalization and removal of the foreign bodies under general anesthesia due to children's lack of cooperation. Fish and chicken bones are the most common swallowed foreign bodies, but an interesting case of a piece of dishwashing steel wool stuck in a child's pharynx is reported in this article.

Case Presentation: A 10-year old boy was presented with a history of dysphagia from five weeks ago. Oral examination did not reveal anything significant. In lateral neck radiographs of the patient, a metal wire was observed in the hypopharyngeal region opposite the 4th and 5th cervical vertebrae. Endoscopic attempts to remove the foreign body were not successful. Therefore, the foreign body was removed from the patient's pharynx in the operating room under general anesthesia. No complications were observed.

Conclusion: Despite the prevalence of swallowing foreign bodies in children, ingestion of a piece of dishwashing steel wool not diagnosed for three weeks was considered interesting to report. Pharyngeal foreign bodies are medical emergencies; therefore, it is particularly important to suspect the presence of a foreign body and perform diagnostic procedures. Keywords: Foreign Body, Ingestion, Children
\end{abstract}

\section{Introduction}

Foreign body ingestion happens at all ages, especially in children (1). Most foreign bodies pass through the gastrointestinal tract without any complications, but pharyngeal foreign body is a medical emergency $(2,3)$. The most common pharyngeal foreign bodies are fish bones, small pieces of food, and plastic toys that often pass with no symptoms, but sometimes they may migrate to the soft tissues of the neck and result in localized infections $(4,5)$. Swallowing of unusual foreign bodies that has been observed in a limited number of cases can cause specific symptoms and complications and may require surgical intervention (6). Factors involved in foreign body complications include the age of the person, the time elapsed since swallowing, the type of foreign body and accompanying factors such as mental retardation $(7,8)$. The present case report pertains to the presentation of a child with a piece of dishwashing steel wool in the pharynx.

\section{Case Presentation}

A 10-year old boy was presented with a history of painful ingestion of liquids and solids from five weeks ago. According to the child and parents, swallowing was associated with throat pain and itching. Oral examination did not reveal anything significant. In lateral neck radiographs of the patient, a metal wire was observed in the hypopharyngeal region opposite the $4^{\text {th }}$ and $5^{\text {th }}$ cervical vertebrae (Figure 1). Endoscopic attempts to remove the foreign body were not successful due to inadequate vision and lack of cooperation from the child. Therefore, he was transferred to the emergency room and the piece of wire was removed from the patient's pharynx under general anesthesia based on Glide Scope guidance using forceps (Figure 2). The patient was discharged the next day in good general conditions and without complications.
Discussion
Swallowing foreign bodies is a common problem in children. Foreign bodies commonly include small pieces of toys, buttons, needles, coins, etc, which mostly pass through the gastrointestinal tract without any complications $(3,4)$. However, foreign bodies that get stuck in the pharynx are of particular importance, 


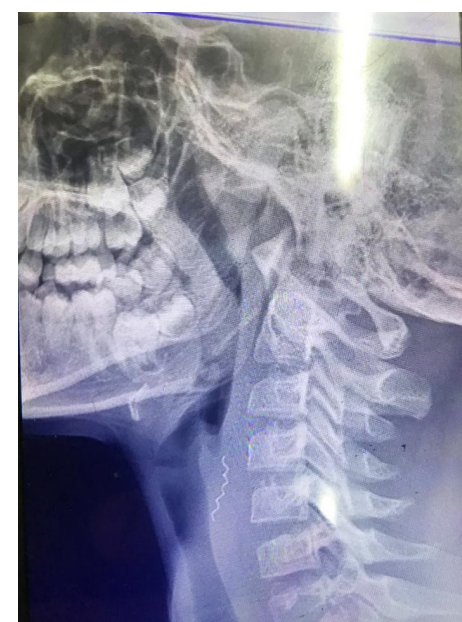

Figure 1. Lateral cervical x-ray shows a piece steel wool (arrow) in the hypopharynx region.

and their diagnosis can be problematic when neither history nor clear clinical signs exist (5). Taking history and careful physical examination can help diagnose the presence of pharyngeal foreign bodies. They can be seen directly in most cases, but sometimes plain radiography, computed tomography (CT) scan scan and even magnetic resonance imaging (MRI) are required (6). Pharyngeal foreign bodies are of particular importance due to their potential complications such as aspiration into the airways, infiltration into the surrounding soft tissues, perforation, inflammatory reaction and infections. Clinical symptoms include painful swallowing, itching, increased salivation, children's refusal to eat and drink and sometimes halitosis. Swallowing unusual objects such as toothbrushes and tools is often seen in people with mental retardation. According to reports, fish and chicken bones are the most common foreign bodies that get stuck in the pharynx $(7,8)$. Regarding the present patient, a piece of dishwashing steel wool that had penetrated into a carrot while washing it got stuck in the hypopharyngeal soft tissue during swallowing. Due to the lack of a clear history, the child who exhibited symptoms of painful swallowing and throat itching received various treatments for infections and allergies for 5 weeks. Since the wire had penetrated into the pharyngeal soft tissue, it could not be observed in direct examination of the pharynx; however, an opaque object was seen in the posterior pharyngeal area on the lateral neck radiograph (Figure 1). Few studies have reported swallowing of unusual foreign bodies; for example, Kim et al reported a case of toothbrush swallowing in a patient with mental retardation (9), and Kurul and Kandogan reported the presence of a gold ring in the throat of a 4-year old child who had been treated for halitosis for 3 years (10). Failure to detect pharyngeal foreign bodies can lead to complications such as infection, cellulitis and abscess formation. Serious complications may occur due to proximity to the airways, esophagus,

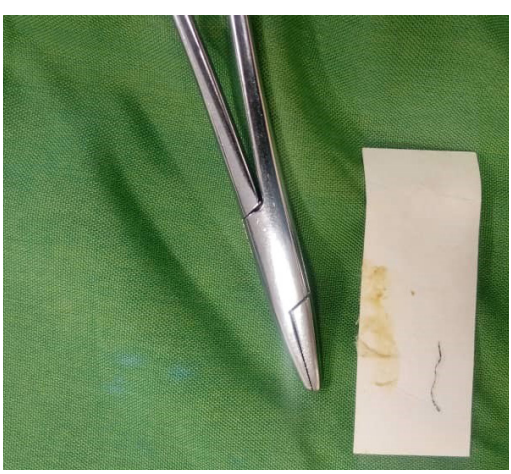

Figure 2. The removed steel wool (arrow).

blood vessels, etc (11-13). The most important challenge in detecting foreign bodies swallowed by children is the lack of a clear history from the child, parents and the caregivers. In the case of our patient, treatment was delayed for 5 weeks because the child and the parents were unaware that the child had swallowed the piece of wire. In cases of suspected foreign body ingestion, diagnostic techniques such as direct vision, endoscopy and plain radiography can be considered, which may be very helpful for detecting radiopaque materials.

\section{Conclusion}

The presence of foreign bodies in the pharynx should be considered in children with symptoms such as painful swallowing, halitosis, and frequent pharyngeal infections. In addition, families should be trained about not washing food materials with tools that have separable and cutting pieces.

\section{Authors' contributions}

PA performed the intervention and MA prepared the manuscript for report.

\section{Ethical Issues}

The authors considered all subjects related ethical issues and the parents of patient signed the consent form.

\section{References}

1. Webb WA. Management of foreign bodies of the upper gastrointestinal tract: update. Gastrointest Endosc 1995; 41(1): 39-51. doi: 10.1016/s0016-5107(95)70274-1.

2. Wai Pak M, Chung Lee W, Kwok Fung H, van Hasselt CA. A prospective study of foreign-body ingestion in 311 children. Int J Pediatr Otorhinolaryngol 2001; 58(1): 37-45. doi: 10.1016/s0165-5876(00)00464-x.

3. Endican S, Garap JP, Dubey SP. Ear, nose and throat foreign bodies in Melanesian children: an analysis of 1037 cases. Int J Pediatr Otorhinolaryngol 2006; 70(9): 1539-45. doi: 10.1016/j.ijporl.2006.03.018.

4. Hesham AKH. Foreign body ingestion: children like to put objects in their mouth. World J Pediatr 2010; 6(4): 301-10. doi: 10.1007/s12519-010-0231-y.

5. Mishra A, Shukla GK, Naresh B. Oropharyngeal 
foreign body. J Laryngol Otol 2000; 114(6): 469-70. doi: 10.1258/0022215001905887.

6. Lai AT, Chow TL, Lee DT, Kwok SP. Risk factors predicting the development of complications after foreign body ingestion. Br J Surg 2003; 90(12): 1531-5. doi: 10.1002/ bjs. 4356 .

7. Watanabe K, Amano M, Nakanome A, Saito D, Hashimoto $\mathrm{S}$. The prolonged presence of a fish bone in the neck. Tohoku J Exp Med 2012; 227(1): 49-52. doi: 10.1620/tjem.227.49.

8. Yang CY, Yang CC. Subjective neck pain or foreign body sensation and the true location of foreign bodies in the pharynx. Acta Otolaryngol 2015; 135(2): 177-80. doi: 10.3109/00016489.2014.973532.

9. Kim YH, Cho SI, Do NY, Park JH. A case of pharyngeal injury in a patient with swallowed toothbrush: a case report. BMC Res Notes 2014; 7: 788. doi: 10.1186/1756-
0500-7-788.

10. Kurul S, Kandogan T. Pharyngeal foreign body in a child persisting for three years. Emerg Med J 2002; 19(4): 361-2. doi: 10.1136/emj.19.4.361.

11. Sharma RC, Dogra SS, Mahajan VK. Oro-pharyngolaryngeal foreign bodies: some interesting cases. Indian J Otolaryngol Head Neck Surg 2012; 64(2): 197-200. doi: 10.1007/s12070-011-0473-6.

12. Yadav SP, Chanda R, Malik $\mathrm{P}$, Chanda S. Ingested nail penetrating the neck in an infant. Int $J$ Pediatr Otorhinolaryngol 2002; 65(2): 159-62. doi: 10.1016/s01655876(02)00149-0.

13. Landis BN, Giger R. An unusual foreign body migrating through time and tissues. Head Face Med 2006; 2: 30. doi: 10.1186/1746-160x-2-30. 